\title{
Le Droit, l'Équité, la Charité : autour de quelques situations judiciaires dans l'œuvre de Shakespeare
}

\section{Henri Suhamy}

Marie-Thérèse Jones-Davies (éd.)

\section{(2) OpenEdition}

Journals

Édition électronique

URL : http://journals.openedition.org/shakespeare/109

DOI : $10.4000 /$ shakespeare.109

ISSN : 2271-6424

Éditeur

Société Française Shakespeare

Édition imprimée

Date de publication : 1 novembre 1980

Pagination : 23-34

\section{Référence électronique}

Henri Suhamy, «Le Droit, l'Équité, la Charité : autour de quelques situations judiciaires dans l'œuvre de Shakespeare ", Actes des congrès de la Société française Shakespeare [En ligne], 2 | 1980, mis en ligne le 01 novembre 2007, consulté le 25 avril 2019. URL : http://journals.openedition.org/shakespeare/109 ; DOI : 10.4000/shakespeare.109 


\section{SOCIÉTÉ FRANC̣AISE SHAKESPEARE}

\section{ACTES DU CONGRĖS 1980}

DIRECTEUR DE LA PUBLICATION

M.T. Jones - Davies

JEAN TOUZOT Libraire - Editeur 38 , rue Saint-Sulpice 75278 PARIS CEDEX 061981 


\section{LE DROIT, L'EQUITE, LA CHARITE : AUTOUR DE QUELQUES SITUATIONS JUDICIAIRES DANS L'OEUVRE DE SHAKESPEARE}

Dans la vie réelle du monde évolué, il n'y a rien de plus théâtral qu'un procès. Rien qui ressemble autant à des personnages de théâtre que ces figures humaines que l'on voit dans le rôle des accusés, des juges, des plaignants, des témoins et des avocats. Rien qui ressemble autant à un décor de théâtre qu'une cour de justice. On y respecte scrupuleusement la règle des trois unités. Y règnent l'artifice, l'illusion, le déguisement, la rhétorique. $Y$ règnent aussi l'étalage impudique des émotions, les incongruités qui déchaînent le rire, l'attente qui provoque l'angoisse, les rebondissements inopinés, les dialogues tendus qui font avancer l'action, les dénouements surprises. Parfois le public est tellement pris au jeu qu'il fait comme au théâtre, il croit à la vérité de tout ce qu'il voit et $\mid$ entend, il manifeste bruyamment son approbation ou sa désapprobation.

Les auteurs dramatiques ont compris depuis longtemps quel parti ils pouvaient tirer du rituel judiciaire, aussi bien dans le domaine de la comédie que dans celui du drame. En France par exemple, on sait qu'il existe, grâce notamment à Jules Moinaux et à son fils Courteline, une tradition inépuisable constituée par le genre appelé Tribunaux Comiques. Mais c'est surtout dans le domaine du drame, et notamment dans celui du mélodrame policier, que l'on voit persévérer cette tradition. On se souvient aujourd'hui encore d'une pièce intitulée Le Procès de Marie Dugan qui fut un des plus durables succès du théâtre de boulevard pendant l'entre-deux-guerres. Je reconnais avoir oublié le nom et la nationalité de son auteur, mais le souvenir si marquant de ce succès populaire, simple exemple parmi beaucoup d'autres, paraît significatif. Comme peut nous paraître aussi significatif le fait que, par exemple, l'adaptation théâtrale du roman de Herman Wouk intitulé en français Ouragan sur le Caine (The Caine Mutiny) ne contient que la scène finale du livre, à savoir le procès des responsables de la mutinerie, qui se termine par un retournement de situation tout à fait spectaculaire.

Ces œuvres utilisent la machine judiciaire comme un 
décor immuable, comme un ensemble de données conventionnelles, les règles d'un jeu que l'on respecte d'autant plus scrupuleusement qu'elles conditionnent le jeu thêâtral luimême. Il arrive bien entendu que ces œuvres contiennent des pointes satiriques; on y dénonce parfois et même souvent l'aveuglement sélectif des juges, volontaire ou involontaire, ou les infirmités morales qui les rendent indignes de leur auguste fonction, voire, comme dans La tête des autres de Marcel Aymé, ou, pour prendre un exemple cinématographique, Le Procès Paradine, d'Alfred Hitchcock, la férocité cannibale de certains procureurs ou magistrats. On y ridiculise aussi la rouerie des uns et des autres, et le formalisme verbeux des professionnels de la chicane, qui délaye les événements les plus poignants et les plus intimes de la vie dans un jargon mystificateur.

Ces œuvres cependant ne remettent pas fondamentalement en cause le principe ni le fonctionnement de la Justice instituée, car, comme on l'a vu, le déroulement même d'une pièce de théâtre ressortissant à ce genre très conventionnel emprunte au cérémonial judiciaire son organisation et son rythme, ses fastes artistiques, sa problématique morale; en effet tout y est fondé sur la notion de culpabilité individuelle, et c'est l'attēnte du châtiment qui procure à la pièce son principal ressort dramatique. Sans vouloir donner à cette constatation un sens agressif et politiquement partial, on peut estimer que le réalisme imitatif du théâtre judiciaire donne au système judiciaire une sorte de justification artistique et morale.

Pour prendre un exemple extrême, la pièce de William Faulkner qui s'appelle Requiem for a Nun, titre qu'on devrait traduire en français par Requiem pour une sour, ou Requiem pour une clotitrée, repose sur une conception plus conformiste de la justice qu'elle ne paraît à première vue. Pourtant cette œuvre prend comme thème la révision idéale et socialement impossible d'un procès déjà jugé; elle nous présente l'envers du décor. Elle soumet le problème de la culpabilité à une analyse rigoureuse, d'une subtilité métaphysiquehors de portée de la machine judiciaire. Mais cette machine judiciaire applique les lois et se fie aux apparences, ce qui semble excusablement humain. Et cette pièce de Faulkner, fondée dans son déroulement sur le système narratif de la révélation progressive et rétrospective, à caractère 
surtout factuel, tout comme dans les romans policiers, reflète en fin de compte une conception juridique pour ne pas dire policière, de la morale, puisque le dramaturge a donné à sa pièce la forme d'une enquête.

La subversion par le théâtre du système judiciaire, on ne peut la trouver véritablement que dans deux types d'œuvres dramatiques : les premières sont celles qui dénoncent brutalement la brutalité des institutions judiciaires ou pseudo-judiciaires, comme certaines/pièces de Brecht, notamment celles qui ne sont pas allégoriques, comme Grand'peur et misères du $3 e$ Reich. Les secondes sont celles qui proposent des visions fantastiquement déformées et symboliques de la justice. L'exemple-type est Le Procès de Kafka, ou plutôt,puisque nous parlons ici de théâtre, l'adaptation qu'en fit André Gide pour Jean-Louis Barrault.

Il n'est pas question ici de postuler implicitement qu'une œuvre d'art n'a d'intérêt que si elle se révèle subversive, anarchiste et révolutionnaire. Une telle conception de l'art en général ou de la littérature n'est pas viable, et même si l'on s'en tient simplement au thème de la Justice, on peut certainement considérer comme respectable la thèse socratique selon laquelle une mauvaise justice vaut mieux que pas de justice du tout, êt qui nous ordonne de toujours obéir aux décisions des juges, même iniques et meurtrières, parce que cette soumission traduit que l'on a confiance dans la civilisation. Peut-être, après tout, ces pièces de théâtre à caractère judiciaire,auxquelles il a été fait allusion, très conventionnelles, ont-elles une influence civilisatrice sur le public.

Tout de même, étant donné que l'appareil judiciaire est une des institutions les plus notoirement imparfaites qui existent, et que la prétention qu'ont les hommes à juger leurs semblables apparaît comme une des manifestations de la démesure terrestre les moins excusables, on attend un peu de la part des auteurs dramatiques qu'ils portent sur la Justice d'ici-bas un regard critique et oblique, et qu'ils fassent par exemple comme Aristophane dans Les guêpes ou Sophocle dans Le roi Oedipe, c'est-à-dire qu'ils organisent pour nous la comédie ou la tragédie de l'arroseur arrosé, je veux dire du juge jugé.

D'autre part on pourrait aussi se demander à quelle catégorie appartient Shakespeare, puisque c'est de lui qu'il 
est question, en principe, même si on semble l'avoir momentanément oublié.

On peut se permettre d'affirmer d'emblée que Shakespeare fait partie de ces grands et rares dramaturges qui subvertissent le rituel judiciaire.

Tout d'abord il faut souligner qu'on trouve dans Shakespeare des dénonciations au premier degré. Quand la Justice tombe entre les mains d'un régime absolutiste, nous dirions aujourd'hui dictatorial ou totalitaire, elle devient une machine à tuer, à étouffer toute velléité d'opposition ou d'alternative. Les prisons, par exemple, les prisons préventives, les prisons oubliettes. On meurt beaucoup dans les prisons shakespeariennes et de façon souvent atroce. Ainsi, dans la seconde partie d'Henry VI, le duc Humphrey est accusé de haute trahison par la faction qui occupe le pouvoir. Afin prétendûment de le protéger contre la colère du peuple et de permettre à l'enquête de suivre son cours en toute sénénité, on le met en prison. En réalité c'est un innocent présumé coupable que l'on fait semblant de traiter avec les égards dus théoriquement à tout coupable présumé innocent. Le lendemain matin on trouve le duc Humphrey étranglé dans sa cellule, le visage violet, les yeux exorbités, qui demandent justice post mortem. Et il y a une justice malgré tout, puisque ce saint homme de cardinal Beaufort, auteur de l'accusation et du crime, sombre dans un remords frénétique et suicidaire. Tout le monde se rappelle la suppression du Prince Arthur dans Le Roi Jean, qui n'échappe à un supplice que pour périr au cours d'une évasion manquée, et dans Richard III l'exécution grandguignolesque de Clarence, l'assassinat des enfants d'Edouard IV. Richard III précisément est fertile en crimes politiques déguisés en opérations judiciaires. On se contentera de rappeler ici l'épisode étonnant du greffier de justice qui, dans un monologue directement adressé au public, vient faire part de sa stupéfaction. On lui a demandé de copier un acte d'accusation dressé contre Lord Hastings, les minutes de son procès, les attendus du jugement et de la condamnation, condamnation à mort bien entendu, avant même que Hastings ait été arrêté. Précisons que Hastings est condamné pour crime de sorcellerie. On comprend que le malheureux greffier éprouve le besoin, comme le barbier du roi Midas, de se débarrasser d'un secret qui lui pèse; mais immergé dans 
la capsule du totalitarisme, il ne trouve personne d'autre à qui confier son secret que le public qui regarde la pièce un siècle plus tard. Des expressions comme parodie de justice ou désordre établi, trop souvent employées de façon abusive et routinière par les polémistes et de ce fait vidées de leur sens, retrouvent ici toute leur force et nous font comprendre que la machine judiciaire, conçue sans doute aux origines de la civilisation pour protéger les faibles contre la force brutale peut devenir un moyen de légitimer la tyrannie et de la répandre en tous lieux grâce à l'ubiquité de ses nervures policières et bureaucratiques. Nous verrons par la suite un autre exemple de la façon dont l'arbitraire politique se sert du système judiciaire pour broyer ses victimes, et nous entendrons demain un exposé de Pierre Sahel sur les prisons politiques dans les cuvres de Marlowe et de Shakespeare.

En attendant il convient d'examiner l'autre aspect de la vision shakespearienne, l'aspect non réaliste quoique tout aussi critique en profondeur, la manière dont Shakespeare enraye et subvertit symboliquement la machine judiciaire, en refusant par exemple de la faire fonctionner normalement.

Ainsi le quatrième acte du Marchand de Venise contient un procès. On n'a pas à revenir ici sur les circonstances de ce procès, sinon peut-être pour souligner que le Tribunal de Venise semble d'abord mettre sa juridiction sourcilleuse et son appareil répressif au service d'une vengeance personnelle. Plus tard grâce à l'avocat Balthazar alias Portia, il se produit un retournement de situation en faveur de l'accusé, ou, plus précisément, du défendeur, et au détriment du plaignant. Voilà, dira-t-on, un exemple de coup de théâtre comme on en voit souvent dans les pièces en forme de procès. En fait, non, la nature de ce coup de théâtre est très différente, car habituellement, la situation change quand des faits, inconnus jusque là, et gardés en réserve par le dramaturge, sont brusquement révélés, ou même se produisent au beau milieu du procès. Ici, dans Le Marchand de Venise, aucun fait nouveau n'apparaît. Balthazar-Portia se contente d'apprendre aux juges leur métier, de leur révéler les articles de loi qu'ils sont censés connaître et appliquer. C'est donc plus qu'un coup de théâtre portant sur des faits, c'est l'institution judiciaire elle-même qui chavire sous 
le poids de sa propre jurisprudence. Des juristes ont étudié le déroulement du procès dans Le Marchand de Venise et en ont déduit que cette histoire était invraisemblable. Nous sommes tous d'accord là-dessus, mais c'est précisément le caractère fantaisiste de cet épisode qui peut avoir en luimême un sens symbolique et satirique. D'autre part l'idée que la loi s'oppose à la loi et les jurisprudences entre elles n'a rien de fantaisiste.

Il existe une autre pièce de Shakespeare qui atteint à une vérité profonde à travers une parabole presque fantastique, la tragi-comédie la plus judiciaire et la plus kafkaïenne qu'il ait écrite : il s'agit évidemment de Mesure pour Mesure. Le titre lui-même est tout à la fois énigmatique, évangélique, et judiciaire. La plupart des scènes se déroulent dans une prison, ou devant un tribunal. Cela se passe à Vienne, une Vienne imaginaire où Johann Strauss n'aurait pas osé intituler une valse Wein Weib und Gesang (en français Aimer,boire et chanter), car l'ordre moral est posé comme un couvercle par une sorte d'Anschluss théocratique. Dans aucune autre pièce de Shakespeare on ne se sent aussi étroitement confiné dans un urbanisme carcéral. Il y est partout question de portes, de clés, de murailles. Le 5e acte se passe en plein air et hors la ville, et cependant cet espace ouvert est lui aussi transformé en tribunal. Tribunal de jugement dernier, il est vrai.

L'événement principal de cette pièce étrange, véritable cauchemar judiciaire, est le procès de Claudio, jeune gentilhomme, condamné à avoir la tête tranchée pour avoir commis le péché de fornication avec sa propre femme, ou du moins avec celle qu'il considère comme son épouse légitime. Il est vrai qu'il manquait à la cérémonie de mariage une ou deux formalités. Le dossier n'est pas complet. II n'en faut pas plus au fanatique qui exerce provisoirement tous les pouvoirs pour faire tomber sur sa victime un châtiment draconien d'autant plus exemplaire qu'il est excessif.

Il est clairement suggéré dans la pièce que toute cette histoire est lourde de signification anagogique, que c'est le péché originell, la souillure de la vie, la condition d'homme, voire même des virtualités d'inceste, détail qui aurait intéressé un autre citoyen de Vienne non dénué de notoriété, qui sont obscurément châtiés chez Claudio. Toụt ceci est bien singulier et intéressant. Mais il y a plus singulier 
encore. Le procès de Claudio. Il n'est question, comme on l'a vu, que de ce procès dans la pièce. Tout le monde en parle, tout le monde se représente en esprit ce drame où s'opposent trois notions, trois forces, la loi, l'équité, la clémence.

Mais ce procès possède en outre une caractéristique très étonnante : l'inexistence. En effet ce procès n'a jamais lieu. Claudio est déjà condamné au moment même de son arrestation. Il n'a même pas droit à une parodie de justice. Juridiquement, il est traité comme un contrevenant passible d'une amende. Seulement l'amende, c'est sa tête. Il a la possibilité de faire appel, ou de faire jouer les influences personnelles dont il dispose, exactement comme s'il était poursuivi pour stationnement interdit. Il est obligé de se défendre par des moyens détournés et à moitié illégaux, puisque, encore une fois, le procès est complètement escamoté.

Nous avons vu que Claudio est déjà condamné au moment de son arrestation. Mais il est plus que condamné, il est culpabilisé. Déjà s'insinue en lui la mentalité du coupable,faite de veulerie, de rouerie, d'insolence vulgaire, de résignation indigne. Et l'imminence de la mort, loin d'avoir l'effet métaphysique et salutaire que lui attribuent les moralistes, ne fait qu'aviver en lui l'instinct de conservation sous sa forme la plus animale.

Nous avons là une démonstration magistrale que l'inculpation précède et provoque le sentiment de culpabilité.

Quant à ce procès évanescent, il n'est nulle part parce qu'il est partout, comme celui de $\mathrm{M}$. $\mathrm{K}$ dans le roman de Kafka. Il est complètement intériorisé, intégré au discours et à la sensibilité de chacun. De même que cette culpabilité diffuse qui est parfois refoulée, mais qui plus souvent encore, ne demande qu'à s'étaler avec complaisance. D'où peut-être ce besoin de tuer des boucs émissaires tout en s'apitoyant sur eux, pour ne pas avoir à pratiquer cette douloureuse opération qu'est la connaissance de soi.

Mesure pour Mesure est une pièce tellement profonde que partie, semble-t-il, d'une réflexion sur la justice, elle aboutit à une réflexion sur la morale. Mais pour en revenir à ce point de départ, ce qu'elle nous apprend sur la conception shakespearienne de la justice reste acquis, ou du moins 
reste un sujet d'exploration extrêmement riche et qui ne saurait se résumer à quelques formules.

On peut aussi hésiter entre deux conceptions globales. $\mathrm{Ou}$ bien on se représente la vision shakespearienne comme essentiellement absurdiste, fondée sur une contemplation esthétique de l'aventure humaine, ou on essaye tout de même d'y trouver un sens, une leçon, exprimée de façon dramatique.

C'est pourquoi on peut reprendre cette trilogie conflictuelle proposée comme titre à ces quelques réflexions, et à laquelle il a été fait allusion tout à l'heure, à savoir le parcours constitué par le Droit, l'Equité et la Charité. Ce triptyque est très important puisqu'il correspond à une problématique fondamentale de la question, tout en se prêtant à une exploitation théâtrale.

Le Droit,c'est l'ensemble des lois et des règlements, ce qui implique le consensus grandiose sur lequel est fondée la civilisation, mais qui implique aussi quelque chose de rigidement écrit et figé, un cérémonial très formel, et aussi une sévérité implacable à laquelle on ne peut se soustraire que de façon clandestine, ou bien, ce qui moralement ne vaut guère mieux, qu'en acceptant le jeu souvent truqué de la procédure. Voir à ce sujet les invectives de Hamlet contre les hommes de loi.

L'équité, notion juridique, c'est le sens inné de la justice qui existe dans l'esprit de tout homme, et qui curieusement a fait l'objet de véritables institutions, puisque ce qu'on appelait jadis en France la Justice de Paix devait s'inspirer de l'équité, indépendamment de toute référence au Droit écrit, Romain ou coutumier. De même on sait qu'en Angleterre la Cour de Chancellerie avait pour fonction de rendre des arrêts fondés sur l'équité, mais n'avait à connaître que des affaires d'ordre privé, des querelles de propriétaires et d'héritiers. La vénalité et les lenteurs de la procédure ont eu raison au 19ème siècle de cette institution stigmatisée par Dickens. Mais l'idéal d'équité n'est pas abandonné par le système judiciaire anglais, puisque des cours d'équité existent toujours.

Quant à la charité, qui prend au tribunal la forme de la clémence, il suffit pour savoir ce qu'elle signifie de se reporter au discours de Portia.

Nous avons vu que Shakespeare nous propose souvent 
des visions abruptes, ou bien mystérieusement illogiques de l'action judiciaire. Il n'est pas impossible cependant que perpendiculairement à ces visions verticales, il nous propose aussi une vision horizontale, un jeu dramatique et dialectique où l'on passerait du Droit à l'Equité, puis de l'Equité à la Charité.

On peut toujours affirmer que dans Le Marchand de Venise Shylock représente le Droit, les amis d'Antonio l'Equité, et Portia la clémence. Rôles assumés respectivement par Angelo, Escalus et Isabella au début de Mesure pour Mesure. Il ne s'agit pas obligatoirement de représentations allégoriques, mais plus simplement et plus explicitement de trois discours successifs : un réquisitoire, une réflexion, une plaidoirie. On ne peut pas cependant en rester là; l'idée d'un déroulement aussi transparent du débat et de l'action est contredite par l'enchevêtrement du texte et des situations. Il est évident que le sens de l'équité, et la charité, vertu théologale et grâce divine, méritent de figurer dans le matériau dramatique. Mais il faut se mettre à la place du dramaturge : faire de l'équité et de la charité des sujets de discours, c'est risquer de faire de la scène du théâtre une ennuyeuse tribune édifiante, une chaire d'où ne seraient émis que des sermons.

D'autre part, montrer l'équité et la charité en action, serait écrire des intrigues trop belles pour être vraisemblebles, des utopies fadement déchiffrables.

Shakespeare semble avoir résolu ce problème de présentation, en plus de ce qui a été dit,à savoir que certains personnages incarnent certains attitudes juridiques avec beaucoup d'authenticité humaine et de tension dramatique. Mais il y a autre chose, et il y a notamment le public, c'est à dire nous.

L'équité est souvent bafouée par la justice officielle. Mais puisque le sens de l'équité est la chose du monde la mieux partagée, comme le bon sens, dont il forme une facette, on peut au moins le trouver dans l'esprit des spectateurs, suscité par certaines situations. Au milieu de celles-ci on est souvent frappé par un détail curieux, déjà entrevu à propos du personnage de Claudio, c'est la résignation des victimes, résignation intellectuelle, sinon physique.

Ainsi le duc de Buckingham, dans Henry VIII, condamné pour crime de lèse-majesté, comme du temps de Tibère. 
Il est accusé d'avoir prononcé une certaine phrase chez lui, il a dit qu'au cas où le roi mourrait sans héritiers légitimes, c'est lui, Buckingham, qui deviendrait l'héritier présomptif. Dénoncé par un serviteur, il est arrêté, jugé, déchu, décapité.

Or personne dans la pièce ne met en question le bienfondé de cette législation tyrannique qui permet au roi de se débarrasser de tous les rivaux et adversaires potentiels de sa dynastie. Buckingham assure lui-même sa défense, avec science et acharnement

Much he spoke

And learnedly for life.

(Il a beaucoup parlé,

et savamment pour sauver sa vie).

Il accepte le système qui devait l'écraser. Il a essayé d'opposer le droit au droit, mais à ce jeu le roi et le Cardinal Wolsey sont plus forts que Shylock, Shylock l'étranger, le paria.

Mais que pense le spectateur qui regarde la pièce? Cette pièce dont le ton reste souvent glacial, distancié. Peut-être le sens de l'équité, c'est-à-dire de la vraie justice, est-il a contrario stimulé par ce spectacle. Le vide crée un appel d'air.

Quant à la clémence, elle est réellement présente. Elle ne se contente pas d'une existence a contrario, en creux. Elle est présente dans le discours, aussi bien le discours théologique que le discours poétique. Elle est également présente en action. Cela entraîne-t-il alors que nous ayons des dénouements utopiques, des dénouements trop beaux pour être crédibles, mais idéalement exemplaires?

Dans un sens oui, mais cet idéalisme est considérablement tempéré par l'ironie des situations. A la fin de Mesure pour Mesure, le Duc exerce son droit de grâce. Le coupable est entièrement pardonné après avoir été pourtant condamné à mort.

Mais attention, à la fin de la pièce, le coupable est en réalité Angelo, c'est-à-dire le juge. C'est le juge qui est jugé, puis grâcié. C'est au cours de cette séance de tribunal en plein air, devant tout le peuple assemblé, qu'Angelo reçoit cette grâce. Et il la reçoit mal, comme une humiliation, comme une gifle. De même dans Le Marchand de Venise, Shylock l'accusateur était condamné puis bénéficiaire d'une grâce partielle, après avoir refusé de l'exercer lui-même. 
Voilà comment en deux occasions Shakespeare nous donne une fin à la fois édifiante et sarcastique. La justice est jugée et bien jugée. La justice est régénérée par une dramaturgie utopique (car ces retournements de dernière minute supposent l'intervention d'un deus ex machina) qui satisfait l'esprit sans endormir sa vigilance. Tel nous apparaît un des paradoxes de ce théâtre visionnaire et lucide.

Henri SUHAMY 